\title{
Subrings of singular cohomology associated to spectra
}

\author{
Geoffrey ML Powell
}

\begin{abstract}
This paper extends the relation established for group cohomology by Green, Hunton and Schuster between chromatic phenomena in stable homotopy theory and certain natural subrings of singular cohomology. This exploits the theory due to Henn, Lannes and Schwartz of unstable algebras over the Steenrod algebra localized away from nilpotents.
\end{abstract}

$55 \mathrm{~S} 10,55 \mathrm{~N} 20 ; 18 \mathrm{~F} 20$

\section{Introduction}

There is a relation between the chromatic filtration of stable homotopy theory and a stratification of the algebraic variety associated to the cohomology of a finite group which was made explicit by the work of Green, Hunton and Schuster [4]. The purpose of this paper is to investigate generalizations of this theory, by exploiting the theory of unstable algebras over the Steenrod algebra for singular cohomology with coefficients in a prime field.

The motivation for the constructions was provided by the Chern ring for group cohomology. A unitary representation $G \rightarrow U(n)$ of a finite group $G$ induces a morphism of classifying spaces $B G \rightarrow B U$ by passage to the infinite unitary group. The induced morphism in singular cohomology $H^{*}(B U) \rightarrow H^{*}(B G)$ is a morphism of rings and the Chern subring $\mathrm{Ch}^{*}(B G) \subset H^{*}(B G)$ is defined to be the subring generated by the image.

The space $B U$ is the connected component of the base point of the space $\Omega^{\infty} K U$ associated to unitary $K$-theory. This suggests a natural definition of a Chern subring associated to an arbitrary spectrum $E$; a further generalization is obtained by considering any topological space in place of $B G$. For a general topological space $X$, there are inclusions of algebras

$$
\mathrm{Ch}_{E}^{*}(X) \hookrightarrow H_{E}^{*}(X) \hookrightarrow H^{*}(X),
$$

where $\mathrm{Ch}_{E}^{*}(X)$ generalizes the Chern subring and $H_{E}^{*}(X)$ is a larger subring defined by a generalization of the construction. 
When singular cohomology is taken with coefficients in a prime field $\mathbb{F}$, these are morphisms of unstable algebras over the Steenrod algebra and are therefore amenable to study from this point of view. Green, Hunton and Schuster studied the ring $\mathrm{Ch}_{E}^{*}(B G)$; for certain generalized cohomology theories, the associated variety is determined in terms of a category related to the Quillen category of elementary abelian $p$-groups associated to the finite group $G$ [4]. The theory of unstable algebras over the Steenrod algebra localized away from nilpotents of Henn, Lannes and Schwartz [6], which is equivalent to working up to $F$-isomorphism, allows a partial generalization of the theory from classifying spaces to more general spaces.

The functor $X \mapsto H_{E}^{*}(X)$ depends upon the topological space $X$ and not only upon the cohomology ring $H^{*}(X)$ as an object of the category $\mathcal{K}$ of unstable algebras over the Steenrod algebra. For $E$ a complex-oriented cohomology theory, there is an algebraic approximation to $H_{E}^{*}(X)$; namely, for an integer $n$ related to the height of the mod $-p$ reduction of the formal group associated to the spectrum $E$, there is a functor $\alpha\langle n\rangle: \mathcal{K} \rightarrow \mathcal{K}$ such that the functor $H_{E}^{*}$ is related to $\alpha\langle n\rangle H^{*}$.

The general result is given in Corollary 6.2.2; it is stated below for the $I_{n}$-complete Johnson-Wilson spectrum, $\widehat{E(n)}$. Recall that a morphism of unstable algebras is an $F$-monomorphism if every element of the kernel is nilpotent.

Theorem A For $n$ a positive integer and $X$ a topological space for which the Hurewicz homomorphism $\left[B \mathbb{F}^{n}, X\right] \rightarrow \operatorname{Hom}_{\mathcal{K}}\left(H^{*}(X), H^{*}\left(B \mathbb{F}^{n}\right)\right)$ is a surjection, there is a natural morphism of unstable algebras over the Steenrod algebra

$$
H_{\widehat{E(n)}}^{*}(X) \rightarrow \alpha\langle n\rangle H^{*}(X)
$$

which is an $F$-monomorphism.

The universal example of the Eilenberg-MacLane spaces shows that this morphism is not an $F$-isomorphism in general. The result of Green, Hunton and Schuster [4] shows that it is an $F$-isomorphism for $X=B G$, the classifying space of a finite group; this uses the work of Hopkins, Kuhn and Ravenel [7] on generalized characters to give the construction of enough elements in the generalized cohomology of a finite group.

The strategy generalizes to the consideration of the Borel cohomology of a $G$-space $X$ to yield the following result.

Theorem B For $G$ a finite group, $X$ a finite $G-C W-$ complex and $n$ a nonnegative integer, there is an $F$-isomorphism of algebras

$$
H_{\overparen{E(n)}}^{*}\left(E G \times_{G} X\right) \cong_{F} \alpha\langle n\rangle H^{*}\left(E G \times_{G} X\right) .
$$


Ackowledgement This paper was motivated by discussions with John Hunton during his visits to Université Paris 13 as 'Professeur Invité' in 2002.

\section{Unstable modules and unstable algebras}

This section provides a rapid overview of the theory of localization of unstable algebras over the Steenrod algebra away from nilpotents. This theory gives the appropriate model for the algebraic variety associated to an unstable algebra. This overview introduces the notation which is used throughout the paper, whereas the reader is referred to the sources for details of the theory.

Let $\mathcal{A}$ denote the Steenrod algebra over $\mathbb{E}$, the prime field of characteristic $p$, which is fixed throughout this paper. The category of unstable modules over $\mathcal{A}$ is denoted by $\mathcal{U}$ and the subcategory of unstable algebras by $\mathcal{K}$ (see Schwartz [12] and Lannes [10]). Singular cohomology is always taken with $\mathbb{F}$-coefficients; it takes values in the category $\mathcal{K}$ of unstable algebras.

\subsection{Localization away from nilpotents}

The theory of Henn, Lannes and Schwartz of localization away from nilpotent unstable modules is a fundamental tool in studying cohomology rings up to $F$-isomorphism (see $[6 ; 9 ; 12])$.

The full subcategory of nilpotent unstable modules is written $\mathcal{N} i l$; localization of the abelian category $\mathcal{U}$ away from the nilpotent modules yields the abelian quotient category $\mathcal{U} / \mathcal{N} i l$. There is the corresponding construction for the category $\mathcal{K} / \mathcal{N} i l$, in which the $F$-isomorphisms become invertible [6, page 1077].

An unstable module $M \in \mathcal{U}$ is naturally the colimit of its finitely generated submodules in $\mathcal{U}$, hence the vector space $\operatorname{Hom}_{\mathcal{U}}\left(M, H^{*}(B V)\right)$ has a natural profinite structure for $V$ an elementary abelian $p$-group; $\operatorname{Hom}_{\mathcal{U}}\left(M, H^{*}(B V)\right)^{\prime}$ denotes the continuous dual. Similarly, an unstable algebra $K \in \mathcal{K}$ is naturally the filtered colimit of its subobjects in $\mathcal{K}$ which are finitely generated as algebras over the Steenrod algebra, hence the set $\operatorname{Hom}_{\mathcal{K}}\left(K, H^{*}(B V)\right)$ has a natural profinite structure.

\subsubsection{Notation}

(1) Let $\mathcal{E}$ denote the category of $\mathbb{F}$-vector spaces and let $\mathcal{E}^{\text {f }}$ denote the full subcategory of finite-dimensional vector spaces.

Algebraic 83 Geometric Topology, Volume 6 (2006) 
(2) Let $\mathcal{F}$ denote the category of functors from $\mathcal{E}^{\mathrm{f}}$ to $\mathcal{E}$ and let $\mathcal{F}_{\omega} \subset \mathcal{F}$ denote the full subcategory of analytic functors. (A functor is analytic if it is the colimit of its finite subobjects, where an object of $\mathcal{F}$ is termed finite if it has a composition series of finite length).

(3) Let $\mathcal{P} \mathcal{S}$ denote the category of profinite sets and continuous morphisms, which are denoted by $\operatorname{Map}_{c}(-,-)$.

(4) Let $\mathcal{G}$ denote the category of functors from $\mathcal{E}^{\mathrm{f}}$ to $\mathcal{P} \mathcal{S}^{\text {op }}$; thus $\mathcal{G}^{\text {op }}$ is equivalent to the category of functors from $\mathcal{E}^{\text {fop }}$ to $\mathcal{P S}$.

(5) (a) Let $f: \mathcal{U} \rightarrow \mathcal{F}$ denote the functor $\left\{V \mapsto \operatorname{Hom}_{\mathcal{U}}\left(-, H^{*}(B V)\right)^{\prime}\right\}$;

(b) let $g: \mathcal{K} \rightarrow \mathcal{G}$ denote the functor $\left\{V \mapsto \operatorname{Hom}_{\mathcal{K}}\left(-, H^{*}(B V)\right)\right\}$.

The functors $f, g$ fit into a commutative diagram in which $\Theta$ denotes the forgetful functor:

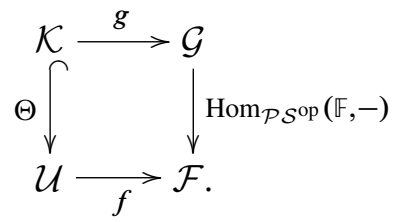

2.1.2 Remark It is frequently convenient to consider the functor $g^{\text {op }:} \mathcal{K}^{\mathrm{op}} \rightarrow \mathcal{G}^{\text {op }}$ in place of $g$; the functor $g^{\text {op }}$ corresponds to a contravariant functor on $\mathcal{K}$ with values in the category of presheaves of profinite sets on $\mathcal{E}^{\text {f }}$.

2.1.3 Definition [6] An object $G \in \mathcal{G}$ is analytic if the functor $\operatorname{Hom}_{\mathcal{P} \mathcal{S}^{\text {op }}}(\mathbb{F}, G)$ is analytic in $\mathcal{F}$. The full subcategory of analytic functors in $\mathcal{G}$ is denoted by $\mathcal{G}_{\omega}$.

The fundamental result of Henn, Lannes, and Schwartz [6] is summarized in the following theorem.

2.1.4 Theorem [6] The functors $f, g$ induce equivalences of categories:

$$
\begin{aligned}
f: \mathcal{U} / \mathcal{N} i l & \cong \\
g: \mathcal{K} / \mathcal{N} i l & \cong \\
\mathcal{F}_{\omega} & \stackrel{\cong}{\rightarrow} \mathcal{G}_{\omega} .
\end{aligned}
$$

2.1.5 Remark The functors which induce the inverse equivalences are induced by the respective right adjoints $a: \mathcal{G} \rightarrow \mathcal{K}$ and $m: \mathcal{F} \rightarrow \mathcal{U}$ to the functors $g: \mathcal{K} \rightarrow \mathcal{G}$ and $f: \mathcal{U} \rightarrow \mathcal{F}($ see $[6])$. 


\section{Subalgebras of singular cohomology}

This section recalls the definition of the generalized Chern subring which is associated to a spectrum, following Green, Hunton and Schuster [4]. For later convenience, various quotients of the set of homotopy classes $[Z, X]$ associated to cohomology functors are introduced using the Hurewicz morphism. The relationships between these functors are explored.

3.0.1 Notation Throughout this section, let $E$ denote a spectrum; the associated $\Omega$-spectrum is denoted $\mathbf{E}_{\bullet}$.

\subsection{Generalized Chern subrings}

Let $\mathcal{T}$ op denote a 'convenient' category of topological spaces; this can be taken to be modelled by the category of simplicial sets.

3.1.1 Definition [4] For $X \in \mathcal{T}$ op a topological space, let

(1) $H_{E}^{*}(X)$ denote the subring of $H^{*}(X)$ generated by cohomology classes represented by (unpointed) morphisms $X \rightarrow \mathbf{E}_{s} \rightarrow K(\mathbb{F}, t)$, for some integers $s, t$

(2) $\mathrm{Ch}_{E}^{*}(X)$ denote the subring of $H^{*}(X)$ generated by cohomology classes represented by (unpointed) morphisms $X \rightarrow \mathbf{E}_{2 s} \rightarrow K(\mathbb{F}, t)$, for some integers $s, t$.

3.1.2 Remark The ring $\mathrm{Ch}_{E}^{*}(X)$ is referred to as the generalized Chern subring; the definition is motivated by the case $X=B G$, the classifying space of a finite group, with $E=K U$ (see [4, Proposition 1.6 and Remark 1.8]).

3.1.3 Proposition For $X \in \mathcal{T}$ op, the algebras $H_{E}^{*}(X)$ and $\mathrm{Ch}_{E}^{*}(X)$ have unique unstable algebra structures over the Steenrod algebra for which the monomorphisms

$$
\mathrm{Ch}_{E}^{*}(X) \hookrightarrow H_{E}^{*}(X) \hookrightarrow H^{*}(X)
$$

are morphisms in $\mathcal{K}$.

Moreover, the associations $X \mapsto H_{E}^{*}(X)$ and $X \mapsto \mathrm{Ch}_{E}^{*}(X)$ are contravariantly functorial in $X$.

Proof Straightforward. 


\subsection{The unstable Hurewicz homomorphism}

Singular cohomology induces a natural unstable Hurewicz transformation

$$
h_{E}: E^{\bullet}(X) \rightarrow \operatorname{Hom}_{\mathcal{K}}\left(H^{*}\left(\mathbf{E}_{\bullet}\right), H^{*}(X)\right) .
$$

3.2.1 Lemma Via the natural inclusions $\mathrm{Ch}_{E}^{*}(X) \hookrightarrow H_{E}^{*}(X) \hookrightarrow H^{*}(X)$, the transformation $h_{E}: E^{\bullet}(X) \rightarrow \operatorname{Hom}_{\mathcal{K}}\left(H^{*}\left(\mathbf{E}_{\bullet}\right), H^{*}(X)\right)$ induces natural transformations:

$$
\begin{aligned}
& h_{E}: E^{\bullet}(X) \rightarrow \operatorname{Hom}_{\mathcal{K}}\left(H^{*}\left(\mathbf{E}_{\bullet}\right), H_{E}^{*}(X)\right) ; \\
& h_{E}: E^{2 \bullet}(X) \rightarrow \operatorname{Hom}_{\mathcal{K}}\left(H^{*}\left(\mathbf{E}_{2 \bullet}\right), \mathrm{Ch}_{E}^{*}(X)\right)
\end{aligned}
$$

Proof Straightforward.

\subsection{Quotients of $[Z, X]$ associated to cohomology theories}

Throughout this section, let $Z, X$ denote spaces in $\mathcal{T} o p$. Proposition 3.3.6 gives an explicit relation between the functors $E^{*}$ and $H_{E}^{*}$. There is an analogous result for the Chern subring $\mathrm{Ch}_{E}^{*}$, by restriction to the even spaces of the $\Omega$-spectrum for $E$.

\subsubsection{Notation}

(1) Let $[Z, X]_{E}$ denote the image of the morphism

$$
[Z, X] \rightarrow \operatorname{Hom}_{E^{*}}\left(E^{*}(X), E^{*}(Z)\right)
$$

induced by $E^{*}$-cohomology and let $q_{E}:[Z, X] \rightarrow[Z, X]_{E}$ denote the canonical surjection.

(2) Let $[Z, X]_{H_{E}}$ denote the image of the morphism

$$
[Z, X] \rightarrow \operatorname{Hom}_{\mathcal{K}}\left(H_{E}^{*}(X), H_{E}^{*}(Z)\right)
$$

induced by the functor $H_{E}^{*}$ and let $q_{H_{E}}:[Z, X] \rightarrow[Z, X]_{H_{E}}$ denote the canonical surjection.

(3) Let $[Z, X]_{H}$ denote the image of the morphism

$$
[Z, X] \rightarrow \operatorname{Hom}_{\mathcal{K}}\left(H^{*}(X), H^{*}(Z)\right)
$$

induced by the functor $H^{*}$ and let $q_{H}:[Z, X] \rightarrow[Z, X]_{H}$ denote the canonical surjection. 
3.3.2 Remark The notation $[-,-]_{H}$ does not conflict with $[-,-]_{E}$, since the image of the morphism $[Z, X] \rightarrow \operatorname{Hom}_{H^{*}}\left(H^{*}(X), H^{*}(Z)\right)$ necessarily takes values in $\operatorname{Hom}_{\mathcal{K}}\left(H^{*}(X), H^{*}(Z)\right)$.

3.3.3 Proposition The constructions $[-,-]_{E}$ and $[-,-]_{H_{E}}$ define functors

$$
\mathcal{T} o p^{\text {op }} \times \mathcal{T} \text { op } \rightarrow \text { Set }
$$

and the morphisms $q_{E}$ and $q_{H_{E}}$ are natural transformations.

Proof Straightforward.

3.3.4 Lemma If the Hurewicz morphism $[Z, X] \rightarrow \operatorname{Hom}_{\mathcal{K}}\left(H^{*}(X), H^{*}(Z)\right)$ is surjective, then there is a canonical isomorphism

$$
[Z, X]_{H} \cong \operatorname{Hom}_{\mathcal{K}}\left(H^{*}(X), H^{*}(Z)\right) .
$$

Proof Tautological.

There is a restriction morphism

$$
\operatorname{Hom}_{\mathcal{K}}\left(H^{*}(X), H^{*}(Z)\right) \rightarrow \operatorname{Hom}_{\mathcal{K}}\left(H_{E}^{*}(X), H^{*}(Z)\right)
$$

induced by the inclusion $H_{E}^{*}(X) \hookrightarrow H^{*}(X)$. However, this need not factor across the inclusion $\operatorname{Hom}_{\mathcal{K}}\left(H_{E}^{*}(X), H_{E}^{*}(Z)\right) \hookrightarrow \operatorname{Hom}_{\mathcal{K}}\left(H_{E}^{*}(X), H^{*}(Z)\right)$ which is induced by $H_{E}^{*}(Z) \hookrightarrow H^{*}(Z)$. When restricting to the image of the Hurewicz morphism, the situation is simpler.

3.3.5 Proposition There is a canonical surjection $p_{H}:[Z, X]_{H} \rightarrow[Z, X]_{H_{E}}$ which factorizes the morphism $q_{H_{E}}:[Z, X] \rightarrow[Z, X]_{H_{E}}$.

Proof For $f: Z \rightarrow X$, the composite $H_{E}^{*}(X) \hookrightarrow H^{*}(X) \stackrel{H^{*}(f)}{\longrightarrow} H^{*}(Z)$ factors canonically across $H_{E}^{*}(f): H_{E}^{*}(X) \rightarrow H_{E}^{*}(Z)$; this defines the map, which is surjective.

3.3.6 Proposition There is a natural commutative diagram

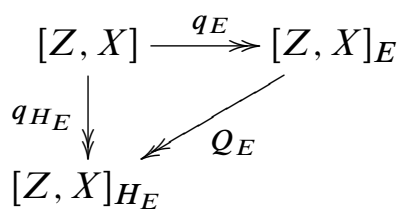

in which the map $Q_{E}$ is surjective.

Proof Straightforward. 


\section{Fundamental properties}

The results of Green, Hunton and Schuster [4] depend on certain fundamental properties of the cohomology theory $E^{*}$ which are developed axiomatically in this section. These results are rephrased here by using the functors $[-,-]_{E}$ and $[-,-]_{H_{E}}$ which were introduced in Section 3.3.

Throughout this section, $E$ denotes a fixed spectrum, $n$ denotes a nonnegative integer, and $Z$ and $X$ denote topological spaces in $\mathcal{T} o p$.

\subsection{The Hurewicz hypotheses}

The following hypothesis allows morphisms in $E$-cohomology to be detected in cohomology.

4.1.1 Hypothesis The Hurewicz hypothesis $\mathbb{H}_{E}(X)$ holds if

$$
h_{E}: E^{\bullet}(X) \rightarrow \operatorname{Hom}_{\mathcal{K}}\left(H^{*}\left(\mathbf{E}_{\bullet}\right), H^{*}(X)\right)
$$

is a monomorphism.

4.1.2 Remark There is an even space variant: the even Hurewicz hypothesis $\mathbb{T}_{E}^{\mathrm{ev}}(X)$ holds if $h_{E}: E^{2 \bullet}(X) \rightarrow \operatorname{Hom}_{\mathcal{K}}\left(H^{*}\left(\mathbf{E}_{2 \bullet}\right), H^{*}(X)\right)$ is a monomorphism.

4.1.3 Example [4, Theorem 4.1] Recall that the spectrum $\widehat{E(n)}$ is the complete version of the Johnson-Wilson theory $E(n)$ [2] and has coefficient ring the completion of $\mathbb{Z}_{(p)}\left[v_{1}, \ldots, v_{n}, v_{n}^{-1}\right]$ with respect to the ideal $I_{n}:=\left(p, v_{1}, \ldots, v_{n-1}\right)$. The $I_{n}$-adically complete Johnson-Wilson spectrum $\widehat{E(n)}$ satisfies the hypothesis $\mathbb{H} \widehat{E(n)}(B V)$, for any $V \in \mathcal{E}^{\mathrm{f}}$.

The following Proposition is the fundamental role of the Hurewicz hypothesis $\mathbb{U}_{E}(Z)$ in separating morphisms in $E$-cohomology. Recall there is a natural transformation $Q_{E}:[Z, X]_{E} \rightarrow[Z, X]_{H_{E}}$ which was introduced in the statement of Proposition 3.3.6.

4.1.4 Proposition Suppose that the Hurewicz hypothesis $\mathbb{H}_{E}(Z)$ holds, then the map

$$
Q_{E}:[Z, X]_{E} \rightarrow[Z, X]_{H_{E}}
$$

is an isomorphism. 
Proof It is sufficient to establish that the map is injective. Let $f_{1}, f_{2}: Z \rightrightarrows X$ be morphisms for which the morphisms $H_{E}^{*}\left(f_{1}\right), H_{E}^{*}\left(f_{2}\right): H_{E}^{*}(X) \rightrightarrows H_{E}^{*}(Z)$ coincide and consider the commutative diagram

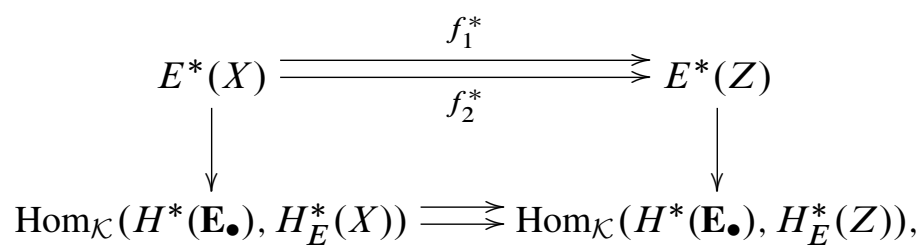

where the bottom arrows are induced by $H_{E}^{*}\left(f_{1}\right), H_{E}^{*}\left(f_{2}\right)$ and hence coincide. The right hand vertical arrow is a monomorphism by hypothesis $\mathbb{U}_{E}(Z)$; the result follows.

4.1.5 Remark Proposition 4.1.4 has an even space variant in which the hypothesis $\mathbb{M}_{E}(Z)$ is replaced by $\mathbb{M}_{E}^{\mathrm{ev}}(Z)$, the subring $H_{E}^{*}(-)$ is replaced by the Chern subring $\mathrm{Ch}_{E}^{*}(-)$ and the statement is restricted to $E^{2 \bullet}(-)$.

\subsection{Height $-n$ detection}

The examples of spectra $E$ which are of interest in applications correspond to complexoriented cohomology theories. For such theories, there is a description of the cohomology $E^{*}(B V)$ of an elementary abelian $p$-group in terms of the associated formal group. The work of Hopkins, Kuhn and Ravenel [7] highlights a fundamental property of a cohomology theory, which is termed height $-n$ detection here. This is the key ingredient to the constructions of this paper.

4.2.1 Notation For $\mathcal{H}$ a functor from $\mathcal{T} o p^{\text {op }}$ to the category of sets, $V$ a finitedimensional $\mathbb{F}$-vector space and $n$ a nonnegative integer, denote by

$$
\delta_{\mathcal{H}}: \mathcal{H}(B V) \rightarrow \mathcal{H}\left(B \mathbb{F}^{n}\right)^{\operatorname{Hom}\left(\mathbb{F}^{n}, V\right)}
$$

the morphism adjoint to the evaluation morphism $\operatorname{Hom}\left(\mathbb{F}^{n}, V\right) \times \mathcal{H}(B V) \rightarrow \mathcal{H}\left(B \mathbb{E}^{n}\right)$.

4.2.2 Definition The spectrum $E$ satisfies the height- $n$ detection property if the morphism

$$
\delta_{E^{*}}: E^{*}(B V) \rightarrow E^{*}\left(B \mathbb{\Vdash}^{n}\right)^{\operatorname{Hom}\left(\mathbb{F}^{n}, V\right)}
$$

is a monomorphism for all $V \in \mathcal{E}^{\mathrm{f}}$.

4.2.3 Remark There is an even space variant: the spectrum $E$ satisfies the even height $-n$ detection property if the above condition is satisfied for the functor $E^{2 *}$. 
4.2.4 Hypothesis Let $E$ be a complex-oriented ring spectrum which satisfies the following conditions:

(1) the coefficient ring $E^{*}$ is a complete, local graded ring with graded maximal ideal $\mathfrak{m}$;

(2) the graded residue field $\kappa:=E^{*} / \mathfrak{m}$ has characteristic $p>0$;

(3) the ring $p^{-1} E^{*}$ is nonzero;

(4) the induced formal group over $E^{*} / \mathfrak{m}$ has height $n$.

4.2.5 Proposition A complex-oriented ring spectrum $E$ which satisfies Hypothesis 4.2.4 satisfies the height $-n$ detection property.

Proof See the proof of [4, Proposition 3.6].

4.2.6 Example The following theories satisfy the height $-n$ detection property:

(1) the $I_{n}$-adically complete Johnson-Wilson theory $\widehat{E(n)}$;

(2) Morava's integral lift of $n$-th Morava $K$-theory, which has coefficient ring $W\left(\mathbb{F}_{p^{n}}\right)\left[u, u^{-1}\right]$, where $W\left(\mathbb{F}_{p^{n}}\right)$ denotes the Witt vectors;

(3) the Lubin-Tate theory $E_{n}$ with coefficient ring

$$
E_{n *} \cong W\left(\mathbb{F}_{p^{n}}\right)\left[\left[w_{1}, \ldots, w_{n-1}\right]\right]\left[u, u^{-1}\right] .
$$

The fundamental consequence of the height $-n$ detection property is given by the following result, which is a formal consequence of the hypothesis.

4.2.7 Proposition Suppose that the spectrum $E$ satisfies the height-n detection property. Then the map

$$
\delta_{[-, X]_{E}}:[B V, X]_{E} \rightarrow\left[B \mathbb{\Vdash}^{n}, X\right]_{E}^{\mathrm{Hom}\left(\mathbb{F}^{n}, V\right)}
$$

is injective, for $V \in \mathcal{E}^{\mathrm{f}}$ an elementary abelian $p$-group.

Proof Straightforward.

Algebraic 83 Geometric Topology, Volume 6 (2006) 


\subsection{Combining the Hurewicz and the height-n detection properties}

The above results combine to give the following Corollary; there is an analogous result when restricting to the even spaces of the $\Omega$-spectrum.

4.3.1 Corollary Suppose that $E$ satisfies the height $n$ detection property and that the Hurewicz hypothesis $\mathbb{U}_{E}\left(B \mathbb{F}^{n}\right)$ is satisfied. Then the map

$$
Q_{E}:[B V, X]_{E} \rightarrow[B V, X]_{H_{E}}
$$

is bijective.

Proof The map $Q_{E}$ is surjective by Proposition 3.3.6, thus we must show that $Q_{E}$ is injective; hence it is sufficient to show that the composite

$$
[B V, X]_{E} \stackrel{Q_{E}}{\longrightarrow}[B V, X]_{H_{E}} \stackrel{\delta_{[-, X]_{H}}}{\longrightarrow}\left[B \mathbb{F}^{n}, X\right]_{H_{E}}^{\mathrm{Hom}\left(\mathbb{F}^{n}, V\right)}
$$

is injective.

Consider the commutative diagram

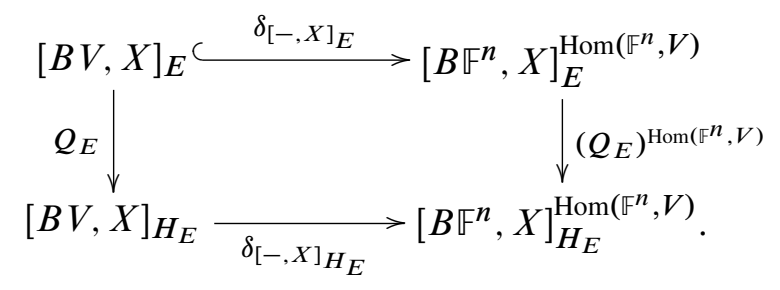

The right hand vertical arrow is a bijection by Proposition 4.1.4, and the top map is injective by Proposition 4.2.7. The result follows.

\section{Filtrations of categories of presheaves}

This section considers natural ways of defining filtrations on certain functor categories. This is presented for general categories of presheaves and is then specialized to the cases of interest, namely certain natural filtrations of the functor category $\mathcal{G}$. The definitions of this section are motivated by the height $-n$ detection property introduced in Section 4.2.

When applied to unstable algebras, the constructions introduce a family of functors $\alpha\langle n\rangle: \mathcal{K} \rightarrow \mathcal{K}$, for $n \in \mathbb{N} \cup\{\infty\}$. These functors take values in nilclosed unstable algebras; they are defined in Section 5.6 and their basic properties are established in Proposition 5.6.2 and Theorem 5.6.3.

Algebraic ${ }^{6} \mathcal{G}$ Geometric Topology, Volume 6 (2006) 


\subsection{Generalities for presheaves}

Throughout this section, let $\mathcal{C}$ denote an (essentially) small category.

\subsubsection{Notation}

(1) Let $\widehat{\mathcal{C}}$ denote the full subcategory $\operatorname{Funct}\left(\mathcal{C}^{\text {op }}\right.$, Set $)$ of presheaves.

(2) Let $\widehat{\mathcal{C}}^{\text {pf }}$ denote the category of presheaves with values in the category $\mathcal{P S}$ of profinite sets.

For clarity of presentation, the following definitions are considered only for the category of presheaves $\widehat{\mathcal{C}}$.

5.1.2 Definition Let $\mathcal{D} \hookrightarrow \mathcal{C}$ be a subcategory and let $F \in \widehat{\mathcal{C}}$ be a presheaf.

(1) For an object $C \in \mathcal{C}$, let $\sim_{\mathcal{D}}$ denote the equivalence relation on $F(C)$ defined by $x \sim_{\mathcal{D}} y$ if and only if $j^{*}(x)=j^{*}(y)$ for each morphism $j: D \rightarrow C$, where $D \in \mathcal{D}$.

(2) Let $\beta_{\mathcal{D}} F(C)$ denote the quotient set $F(C) / \sim_{\mathcal{D}}$.

\subsubsection{Lemma}

(1) The association $C \mapsto \beta_{\mathcal{D}} F(C)$ defines a presheaf $\beta_{\mathcal{D}} F$.

(2) The association $\beta_{\mathcal{D}}$ defines a functor $\beta_{\mathcal{D}}: \widehat{\mathcal{C}} \rightarrow \widehat{\mathcal{C}}$.

(3) There is a natural surjection $1_{\widehat{\mathcal{C}}} \rightarrow \beta_{\mathcal{D}}$.

(4) For $D$ an object of $\mathcal{D}, \beta_{\mathcal{D}} F(D)=F(D)$.

(5) The functor $\beta_{\mathcal{D}}$ depends only upon the full subcategory generated by $\mathcal{D}$.

Proof Straightforward.

The following result has important consequences:

5.1.4 Proposition Let $\mathcal{D} \hookrightarrow \mathcal{C}$ be a full small subcategory, and let $\beta_{\mathcal{D}}: \widehat{\mathcal{C}} \rightarrow \widehat{\mathcal{C}}$ denote the induced functor. Then

(1) $\beta_{\mathcal{D}}$ preserves surjections;

(2) $\beta_{\mathcal{D}}$ preserves injections;

(3) $\beta_{\mathcal{D}}$ commutes with finite products: namely, for presheaves $F, G \in \widehat{\mathcal{C}}$, there is a canonical isomorphism $\left(\beta_{\mathcal{D}} F\right) \times\left(\beta_{\mathcal{D}} G\right) \cong \beta_{\mathcal{D}}(F \times G)$. 
Proof The first statement is a consequence of the definition of $\beta_{\mathcal{D}}$ as a natural quotient. The second statement is essentially formal: suppose that $\gamma: F \hookrightarrow G$ is an inclusion of presheaves and let $x, y \in F(C)$ represent distinct elements in $\beta_{\mathcal{D}} F$. It is necessary to show that $\gamma_{C} x$ and $\gamma_{C} y$ represent distinct elements in $\beta_{\mathcal{D}} G(C)$. By definition, there exists a morphism $f: D \rightarrow C$ with $D \in \mathcal{D}$ for which $f^{*}(x) \neq f^{*}(y)$ in $F(D)$. Hence, the images $\gamma_{D} f^{*}(x)$ and $\gamma_{D} f^{*}(y)$ are distinct in $G(D)$, since $\gamma_{D}$ is an injection. Functoriality gives $\gamma_{D} f^{*}=f^{*} \gamma_{C}$, so the result follows.

Let $F, G \in \widehat{\mathcal{C}}$ be presheaves and let $C$ be an object in $\mathcal{C}$. The result follows from the observation that $\left(x_{1}, y_{1}\right) \sim_{\mathcal{D}}\left(x_{2}, y_{2}\right)$ in $(F \times G)(C)$ if and only if $x_{1} \sim_{\mathcal{D}} x_{2}$ and $y_{1} \sim \mathcal{D} y_{2}$.

\subsection{A fundamental property of the construction $\beta_{\mathcal{D}}$}

The notation of the previous section is maintained.

5.2.1 Proposition Let $v: F \rightarrow G$ be a morphism of presheaves in $\widehat{\mathcal{C}}$ which factors as $v_{\mathcal{D}}: \beta_{\mathcal{D}} F \rightarrow G$. Then $v_{\mathcal{D}}$ is a monomorphism of presheaves if and only if the restriction $\left.\left.F\right|_{\mathcal{D}} \rightarrow G\right|_{\mathcal{D}}$ is a monomorphism.

Proof The forward implication is straightforward, hence consider the converse. If $v_{\mathcal{D}}: \beta_{\mathcal{D}} F \rightarrow G$ is not a monomorphism, then there exist sections $x, y \in F(C)$, for some object $C$ of $\mathcal{C}$ such that $x$ and $y$ represent distinct elements in $\beta_{\mathcal{D}} F(C)$ and $v(x)=v(y)$. The first condition implies that there exists a morphism $j: D \rightarrow C$ in $\mathcal{C}$, where $D$ is an object of $\mathcal{D}$, such that $j^{*} x \neq j^{*} y$ in $F(D)=\beta_{\mathcal{D}} F(D)$. It follows that the morphism $F(D) \rightarrow G(D)$ is not a monomorphism, by naturality, which implies the required result.

\subsection{Presheaves of profinite sets}

The general application of these constructions to unstable modules requires the consideration of presheaves with values in profinite sets. (For the applications of this paper, it would be sufficient to restrict to presheaves with values in finite sets).

5.3.1 Proposition The functor $\beta_{\mathcal{D}}: \widehat{\mathcal{C}} \rightarrow \widehat{\mathcal{C}}$ induces a functor on the category of presheaves with values in profinite sets, $\beta_{\mathcal{D}}: \widehat{\mathcal{C}}^{\text {pf }} \rightarrow \widehat{\mathcal{C}}^{\text {pf }}$.

Proof Straightforward.

Algebraic $6 \mathcal{G}$ Geometric Topology, Volume 6 (2006) 


\subsection{Presheaves on finite-dimensional vector spaces}

The motivating example is the category of presheaves of profinite sets on the category of finite-dimensional vector spaces over the prime field $\mathbb{F}$.

5.4.1 Notation For $n$ a nonnegative integer or $\infty$, let $\mathcal{E}^{\mathrm{f}}\langle n\rangle$ denote the full subcategory of $\mathcal{E}^{\mathrm{f}}$ with set of objects $\left\{\mathbb{F}^{t} \mid t \leq n\right\}$.

Recall that $\mathcal{G}$ denotes the category of functors from $\mathcal{E}^{\mathrm{f}}$ to $\mathcal{P} \mathcal{S}^{\text {op }}$, so that $\mathcal{G}^{\text {op }}$ is equivalent to a category of presheaves on $\mathcal{E}^{\mathrm{f}}$ with values in profinite sets. The general definitions for presheaves yield the following functors, in which attention must be paid to the variance.

5.4.2 Definition For $n$ a nonnegative integer or $\infty$, let $\beta\langle n\rangle: \mathcal{G}^{\text {op }} \rightarrow \mathcal{G}^{\text {op }}$ denote the functor $\beta_{\mathcal{E}^{\mathrm{f}}\langle n\rangle}$ defined on the category of presheaves on $\mathcal{E}^{\mathrm{f}}$ with values in profinite sets.

By construction, there is a canonical surjection in $\mathcal{G}^{\text {op }}, G \rightarrow \beta\langle n\rangle G$ for each $G \in \mathcal{G}$.

5.4.3 Proposition For $n$ a nonnegative integer:

(1) the functor $\beta\langle\infty\rangle$ is equivalent to the identity functor;

(2) there is a natural surjection $\beta\langle n+1\rangle \rightarrow \beta\langle n\rangle$ of endofunctors on $\mathcal{G}^{\text {op }}$ which occurs in the diagram of natural transformations

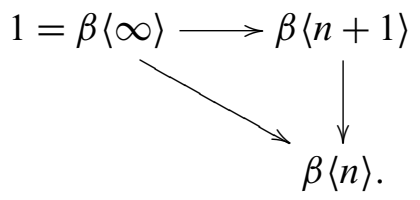

Proof The first statement is clear; to prove the second, it is sufficient to show that the natural transformation $1 \rightarrow \beta\langle n\rangle$ factorizes naturally across $\beta\langle n+1\rangle$.

Let $G$ be an object of $\mathcal{G}^{\text {op }}$ and consider sections $s_{0}, s_{1} \in G(V)$ such that, for any morphism $j: \mathbb{F}^{n+1} \rightarrow V$, the sections $j^{*} s_{0}$ and $j^{*} s_{1}$ are equal in $G\left(\mathbb{F}^{n+1}\right)$. Consider a morphism $k: \mathbb{F}^{n} \rightarrow V$; there exists a factorization $\mathbb{F}^{n} \rightarrow \mathbb{F}^{n+1} \stackrel{j}{\rightarrow} V$ across a morphism $j$. It follows that $k^{*} s_{0}=k^{*} s_{1}$, which implies that the morphism $1 \rightarrow \beta\langle n\rangle$ factors across $1 \rightarrow \beta\langle n+1\rangle$, as required.

5.4.4 Example Let $\operatorname{Hom}(-, W)$ denote the object of $\mathcal{G}^{\text {op }}$ given by $V \mapsto \operatorname{Hom}(V, W)$ (forgetting the abelian structure) and let $n$ be a nonnegative integer. Linearity implies that $\beta\langle n\rangle \operatorname{Hom}(-, W) \cong \operatorname{Hom}(-, W)$, so that the filtration of $\operatorname{Hom}(-, W)$ provided by $\beta\langle n\rangle$ as $n$ varies is constant. 
5.4.5 Proposition For $n$ a nonnegative integer or $\infty$, the functor $\beta\langle n\rangle$ restricts to a functor $\beta\langle n\rangle: \mathcal{G}_{\omega}^{\mathrm{op}} \rightarrow \mathcal{G}_{\omega}^{\mathrm{op}}$.

Proof Consider an object $G \in \mathcal{G}^{\text {op }}$ which corresponds to an analytic functor. The canonical surjection $G \rightarrow \beta\langle n\rangle G$ induces a monomorphism in $\mathcal{F}$ under the functor $\operatorname{Map}_{c}(-, \mathbb{F})$. This identifies $\operatorname{Map}_{c}(\beta\langle n\rangle G, \mathbb{F})$ as a subobject of an analytic functor by the hypothesis on $G$, hence $\beta\langle n\rangle G$ is analytic, as required.

\subsection{Functors induced from $\mathcal{E}^{\mathrm{f}}\langle d\rangle$}

The theory of Henn, Lannes and Schwartz [6] provides an elegant treatment of unstable algebras of transcendence degree $d$, for $d$ a nonnegative integer. This section shows that the functors $\beta\langle n\rangle$ restrict well to this theory.

5.5.1 Notation For $d$ a nonnegative integer, let $\mathcal{G}\langle d\rangle$ denote the category of functors from $\mathcal{E}^{\mathrm{f}}\langle d\rangle$ to $\mathcal{P} \mathcal{S}^{\mathrm{op}}$, so that $\mathcal{G}\langle d\rangle^{\mathrm{op}}$ is the category of presheaves on $\mathcal{E}^{\mathrm{f}}\langle d\rangle$ with values in profinite sets. Let $e_{d}: \mathcal{G}^{\mathrm{op}} \rightarrow \mathcal{G}\langle d\rangle^{\mathrm{op}}$ denote the canonical restriction functor.

The importance of the category $\mathcal{G}\langle d\rangle$ is given by its connection with the category $\mathcal{K}$ [6]. Let $\mathcal{K}_{d}$ denote the full subcategory of $\mathcal{K}$ the objects of which have underlying algebra of transcendence degree at most $d$; the category $\mathcal{K}_{d}$ is closed under $F$-isomorphism and the following result holds.

5.5.2 Theorem [6, Theorem II.2.8] The functor $g: \mathcal{K} \rightarrow \mathcal{G}$ induces an equivalence of categories $\mathcal{K}_{d} / \mathcal{N} i l \cong \mathcal{G}\langle d\rangle$.

The category $\mathcal{G}\langle d\rangle^{\text {op }}$ is equivalent to the category of profinite right $\operatorname{End}\left(\mathbb{F}^{d}\right)$-sets $[6$, Section II.2]. The functor $e_{d}$ identifies with the evaluation functor on $\mathbb{F}^{d}$ and admits a left adjoint $i_{d}: \mathcal{G}\langle d\rangle^{\text {op }} \rightarrow \mathcal{G}^{\text {op }}$ which is given by $S \mapsto S\left(\mathbb{F}^{d}\right) \times_{\operatorname{End}\left(\mathbb{F}^{d}\right)} \operatorname{Hom}\left(-, \mathbb{F}^{d}\right)$. A fundamental property is the following result.

5.5.3 Lemma [6, Key Lemma, Lemma 2.1] Let $G \in \mathcal{G}^{\text {op }}$ be a presheaf of profinite sets on $\mathcal{E}^{\mathrm{f}}$. Then the canonical morphism $i_{d} e_{d} G \rightarrow G$ is a monomorphism.

5.5.4 Notation Let $\beta\langle n\rangle: \mathcal{G}\langle d\rangle^{\mathrm{op}} \rightarrow \mathcal{G}\langle d\rangle^{\text {op }}$ denote the analogue of the functor $\beta\langle n\rangle: \mathcal{G}^{\mathrm{op}} \rightarrow \mathcal{G}^{\mathrm{op}}$.

The following result gives a criterion for the natural transformation $1 \rightarrow \beta\langle n\rangle$ to yield an isomorphism when evaluated on an object. 
5.5.5 Proposition For nonnegative integers $n$ and $d$ and $S \in \mathcal{G}\langle d\rangle^{\text {op }}$ a profinite presheaf on $\mathcal{E}^{\mathrm{f}}\langle d\rangle$, there is a natural isomorphism

$$
i_{d}(\beta\langle n\rangle S) \stackrel{\cong}{\rightarrow} \beta\langle n\rangle\left(i_{d} S\right) .
$$

In particular for $n \geq d$, there is an identification $\beta\langle n\rangle\left(i_{d} S\right)=i_{d} S$.

Proof Write $G$ for $i_{d} S$, then there is a natural surjection $G \rightarrow \beta\langle n\rangle G$. From the definitions, it is clear that there is an identification $e_{d}(\beta\langle n\rangle G)=\beta\langle n\rangle\left(e_{d} G\right)$. The composite

$$
i_{d} \beta\langle n\rangle\left(e_{d} G\right) \stackrel{\cong}{\rightarrow} i_{d} e_{d}(\beta\langle n\rangle G) \hookrightarrow \beta\langle n\rangle G
$$

is a monomorphism by Lemma 5.5.3. This implies the first statement; the second follows immediately.

\subsection{The functor $\alpha\langle n\rangle$ and unstable algebras}

The functor $\beta\langle n\rangle^{\text {op }}$ induces a functor on the category $\mathcal{K} / \mathcal{N}$ il by Theorem 2.1.4; composing with the functors involved in the nil-localization $\mathcal{K} \leftrightarrows \mathcal{K} / \mathcal{N}$ il yields a functor on the category $\mathcal{K}$ of unstable algebras. (Note that the variance considerations for the functor $g$ require passage to the opposite category.) The reader is referred to Henn, Lannes and Schwartz [6] for the relevant definitions concerning the nillocalization of unstable algebras.

5.6.1 Notation For $K \in \mathcal{K}$ an unstable algebra and $n$ a nonnegative integer or $\infty$, write $\alpha\langle n\rangle K$ for the nilclosed unstable algebra which is associated to the functor $\beta\langle n\rangle^{\mathrm{op}} g K$.

The properties of the functor $\beta\langle n\rangle: \mathcal{G}^{\mathrm{op}} \rightarrow \mathcal{G}^{\mathrm{op}}$ imply the following properties of the induced functor on unstable algebras.

5.6.2 Proposition For $n$ a nonnegative integer or $\infty$ and $K \in \mathcal{K}$ an unstable algebra, the following properties hold.

(1) The association $\alpha\langle n\rangle: K \mapsto \alpha\langle n\rangle K$ defines a functor $\alpha\langle n\rangle: \mathcal{K} \rightarrow \mathcal{K}$ with values in nilclosed unstable algebras.

(2) The functor $\alpha\langle\infty\rangle$ identifies with the nilclosure functor.

(3) The functor $\alpha\langle n\rangle: \mathcal{K} \rightarrow \mathcal{K}$ preserves injections. 
(4) There is a commutative diagram of natural monomorphisms

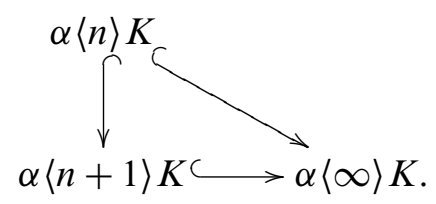

Proof The result is a direct translation of Proposition 5.4.3 using Proposition 5.4.5 to apply Theorem 2.1.4.

The behaviour of the functors $\alpha\langle n\rangle$ on unstable algebras of finite transcendence degree is described by the following result.

5.6.3 Theorem If $K \in \mathcal{K}_{d}$ is an unstable algebra of transcendence degree $d$ then $\alpha\langle n\rangle K$ is an unstable algebra of transcendence degree at most $d$. Moreover, if $n \geq d$, then $\alpha\langle n\rangle K$ identifies with the nilclosure of $K$.

Proof The result is an immediate consequence of Proposition 5.5.5.

\section{On the varieties associated to the rings $H_{E}^{*}(-)$}

The study of the algebraic variety associated to a ring $H_{E}^{*}(X)$ is essentially equivalent to the study of the functor $g H_{E}^{*}(X) \in \mathcal{G}$. The results of this section are stated for the rings $H_{E}^{*}$; there are analogous results for the rings $\mathrm{Ch}_{E}^{*}$.

\subsection{Preliminaries}

To generalize the results of Green, Hunton and Schuster [4] requires realizing morphisms between certain unstable algebras topologically. This uses part of the theory of unstable algebras.

Recall the nonlinear injectivity property of the objects $H^{*}(B V) \in \mathcal{K}$.

6.1.1 Proposition [12, Corollary 3.8.7] For a monomorphism $i: K \hookrightarrow L$ and a morphism $\phi: K \rightarrow H^{*}(B V)$ in $\mathcal{K}$, there exists an extension $\tilde{\phi}: L \rightarrow H^{*}(B V)$ in $\mathcal{K}$ such that $\phi=\widetilde{\phi} \circ i$.

6.1.2 Corollary Let $X$ be a topological space for which the Hurewicz morphism $[B V, X] \rightarrow \operatorname{Hom}_{\mathcal{K}}\left(H^{*}(X), H^{*}(B V)\right)$ is surjective, where $V \in \mathcal{E}^{\mathrm{f}}$ is an elementary abelian $p$-group. Then there are canonical bijections:

$$
\begin{aligned}
& {[B V, X]_{H} } \cong \operatorname{Hom}_{\mathcal{K}}\left(H^{*}(X), H^{*}(B V)\right) ; \\
& {[B V, X]_{H_{E}} \cong \operatorname{Hom}_{\mathcal{K}}\left(H_{E}^{*}(X), H_{E}^{*}(B V)\right) . }
\end{aligned}
$$


Proof The first bijection is a special case of Lemma 3.3.4. To prove the second, it is sufficient to show that the functor $H_{E}^{*}$ induces a surjection from $[B V, X]$ onto $\operatorname{Hom}_{\mathcal{K}}\left(H_{E}^{*}(X), H_{E}^{*}(B V)\right)$.

A morphism $\alpha: H_{E}^{*}(X) \rightarrow H_{E}^{*}(B V)$ of unstable algebras extends to a morphism $H^{*}(X) \rightarrow H^{*}(B V)$ by Proposition 6.1.1. Hence, $\alpha$ is in the image of the morphism $[B V, X]_{H} \rightarrow[B V, X]_{H_{E}}$ and the result follows by the first statement.

The hypothesis on the surjectivity of the Hurewicz homomorphism is satisfied, for example, if the topological space $X$ satisfies the hypotheses of the following result.

6.1.3 Theorem [10] Let $X$ be a connected topological spaces which is of finite type, nilpotent and such that $\pi_{1}(X)$ is finite. Then the Hurewicz morphism

$$
[B V, X] \rightarrow \operatorname{Hom}_{\mathcal{K}}\left(H^{*}(X), H^{*}(B V)\right)
$$

is an isomorphism.

\subsection{Algebraic approximation to the rings $H_{E}^{*}$}

The theory of the Chern subring for group cohomology [4] admits a partial generalization under suitable hypotheses; the general result is stated below in Theorem 6.2.1.

6.2.1 Theorem Suppose that there exists a positive integer $n$ such that

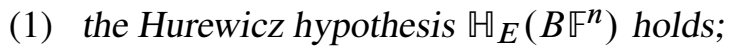

(2) $E$ satisfies the height $-n$ detection property.

Then for $X$ a topological space for which the morphism

$$
[B V, X] \rightarrow \operatorname{Hom}_{\mathcal{K}}\left(H^{*}(X), H^{*}(B V)\right)
$$

is surjective, the natural transformation $1 \rightarrow \beta\langle n\rangle$ induces an isomorphism of functors in $\mathcal{G}^{\text {op }}$

$$
g^{\mathrm{op}} H_{E}^{*}(X) \stackrel{\cong}{\rightarrow} \beta\langle n\rangle g^{\mathrm{op}} H_{E}^{*}(X)
$$

Proof The natural transformation $1 \rightarrow \beta\langle n\rangle$ is surjective, hence it is sufficient to prove injectivity. Let $V \in \mathcal{E}^{\mathrm{f}}$ be an elementary abelian $p$-group and let $y_{1}, y_{2} \in g H_{E}^{*}(X)(V)$ be elements such that $j^{*} y_{1}=j^{*} y_{2}$, for all morphisms $j: \mathbb{F}^{n} \rightarrow V$. The definition of the functor $g: \mathcal{K}^{\mathrm{op}} \rightarrow \mathcal{G}^{\mathrm{op}}$ together with Corollary 6.1.2 imply that the elements $y_{i}$ are represented by morphisms $H_{E}^{*}\left(f_{i}\right)$ for $f_{i} \in[B V, X], i \in\{1,2\}$. 
The hypothesis upon the elements $y_{i}$ implies that the two composites in the following diagram coincide:

$$
H_{E}^{*}(X) \stackrel{f_{1}^{*}}{\underset{f_{2}^{*}}{\longrightarrow}} H_{E}^{*}(B V) \stackrel{j^{*}}{\longrightarrow} H_{E}^{*}\left(B \mathbb{\Vdash}^{n}\right),
$$

for all morphisms $j: \mathbb{F}^{n} \rightarrow V$. The morphisms $E^{*}\left(f_{1}\right), E^{*}\left(f_{2}\right): E^{*}(X) \rightrightarrows E^{*}(B V)$ coincide by Corollary 4.3.1; hence Proposition 4.1.4 implies that the elements $y_{1}$ and $y_{2}$ are equal. It follows that the canonical morphism $g^{\mathrm{op}} H_{E}^{*}(X) \rightarrow \beta\langle n\rangle g^{\mathrm{op}} H_{E}^{*}(X)$ is a monomorphism, as required.

6.2.2 Corollary Let $E$ be a spectrum which satisfies the hypotheses of Theorem 6.2.1, and let $X$ be a topological space for which the morphism

$$
[B V, X] \rightarrow \operatorname{Hom}_{\mathcal{K}}\left(H^{*}(X), H^{*}(B V)\right)
$$

is surjective. Then there is a canonical morphism

$$
H_{E}^{*}(X) \rightarrow \alpha\langle n\rangle H^{*}(X)
$$

in $\mathcal{K}$ which induces a monomorphism in $\mathcal{K} / \mathcal{N} i l$.

Proof Recall that the functor $\alpha\langle\infty\rangle$ coincides with the nil-localization functor and that there is a canonical morphism $H_{E}^{*}(X) \rightarrow \alpha\langle\infty\rangle H_{E}^{*}(X)$ which induces an isomorphism in $\mathcal{K} / \mathcal{N} i l$. There is a commutative diagram

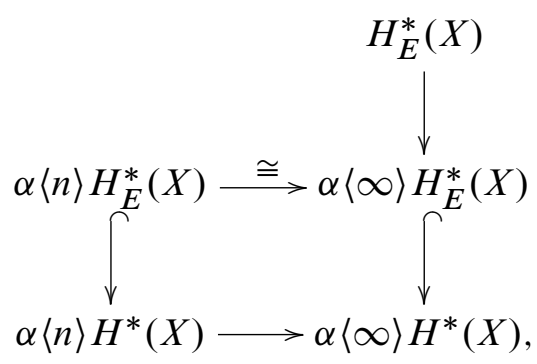

in which the top horizontal morphism is an isomorphism by Theorem 6.2.1. The left hand vertical morphism is a monomorphism, since $\alpha\langle n\rangle: \mathcal{K} \rightarrow \mathcal{K}$ preserves injections. The result follows.

\subsubsection{Remark}

(1) In general, the morphism $H_{E}^{*}(X) \rightarrow \alpha\langle n\rangle H^{*}(X)$ is not a monomorphism, since the unstable algebra $H_{E}^{*}(X)$ need not be nilclosed. For example, consider the case $E=K U$, unitary $K$-theory and $X=\mathbb{C} P^{n}$. 
(2) It is not clear that the topological functor $X \mapsto H_{E}^{*}(X)$ extends in general to a functor $\mathcal{K} \rightarrow \mathcal{K} / \mathcal{N} i l$, especially in the light of Example 6.4.1 below.

\subsection{Separation}

It is natural to seek a class of spaces for which the natural morphism from $H_{E}^{*}(X)$ to $\alpha\langle n\rangle H^{*}(X)$ is an isomorphism in $\mathcal{K} / \mathcal{N} i l$. For example, the results of Green, Hunton and Schuster [4] concern classifying spaces of finite groups; in this case, Hopkins, Kuhn and Ravenel [7] provide a construction of enough elements in $E^{*}$-cohomology so as to obtain equality up to $F$-isomorphism.

6.3.1 Lemma Suppose that there exists a positive integer $n$ such that the Hurewicz

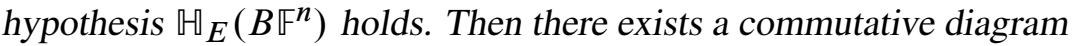

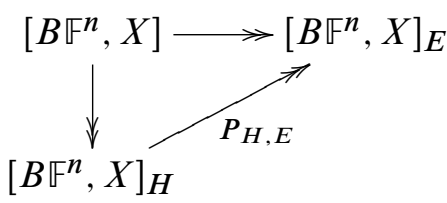

of surjections.

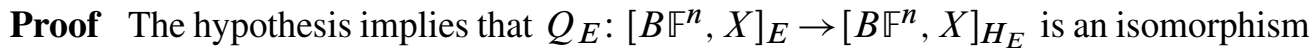
by Proposition 4.1.4. The result follows from the commutative diagram

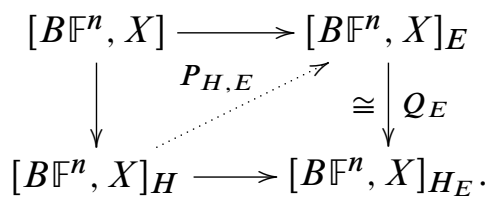

The following result gives a separation criterion which is sufficient to prove equality in $\mathcal{K} / \mathcal{N}$ il between the unstable algebras $H_{E}^{*}(X)$ and $\alpha\langle n\rangle H^{*}(X)$, under suitable hypotheses.

6.3.2 Proposition Suppose that there exists a positive integer $n$ such that

(1) the Hurewicz hypothesis $\mathbb{H}_{E}\left(B \mathbb{\mathbb { F }}^{n}\right)$ holds;

(2) $E$ satisfies the height $-n$ detection property.

Let $X \in \mathcal{T}$ op be a space for which the morphism

$$
\left[B \mathbb{F}^{n}, X\right] \rightarrow \operatorname{Hom}_{\mathcal{K}}\left(H^{*}(X), H^{*}\left(B \mathbb{F}^{n}\right)\right)
$$

Algebraic ${ }^{6} \mathcal{G}$ Geometric Topology, Volume 6 (2006) 
is surjective.

Suppose that the morphism

$$
P_{H, E}:\left[B \mathbb{\Vdash}^{n}, X\right]_{H} \rightarrow\left[B \mathbb{\Vdash}^{n}, X\right]_{E}
$$

is an injection. Then there is an isomorphism $H_{E}^{*}(X) \cong \mathcal{K} / \mathcal{N} i l \alpha\langle n\rangle H^{*}(X)$ in $\mathcal{K} / \mathcal{N} i l$.

Proof The morphism $H_{E}^{*}(X) \rightarrow \alpha\langle n\rangle H^{*}(X)$ corresponds to a morphism

$$
\beta\langle n\rangle g^{\mathrm{op}} H^{*}(X) \rightarrow g^{\mathrm{op}} H_{E}^{*}(X),
$$

and it is sufficient to show that this is a monomorphism under the given hypothesis. It is sufficient to show that the morphism $g^{\text {op }} H^{*}(X) \rightarrow g^{\text {op }} H_{E}^{*}(X)$ is a monomorphism when evaluated on $\mathbb{F}^{n}$ by Proposition 5.2.1.

The hypothesis upon $P_{H, E}$ implies that the morphism $\left[B \mathbb{F}^{n}, X\right]_{H} \rightarrow\left[B \mathbb{F}^{n}, X\right]_{H_{E}}$ is an isomorphism. Moreover, the surjectivity of the Hurewicz monomorphism implies that there is an isomorphism $\left[B \mathbb{F}^{n}, X\right]_{H} \cong \operatorname{Hom}_{\mathcal{K}}\left(H^{*}(X), H^{*}\left(B \mathbb{F}^{n}\right)\right)$ and an isomorphism $\left[B \mathbb{E}^{n}, X\right]_{H_{E}} \cong \operatorname{Hom}_{\mathcal{K}}\left(H_{E}^{*}(X), H_{E}^{*}\left(B \mathbb{F}^{n}\right)\right)$. Hence the natural morphism

$$
g^{\mathrm{op}} H^{*}(X)\left(\mathbb{F}^{n}\right) \cong g^{\mathrm{op}} H_{E}^{*}(X)\left(\mathbb{F}^{n}\right)
$$

is an isomorphism, as required.

\subsection{An example for $E=K U$}

The following example shows that the monomorphism in $\mathcal{K} / \mathcal{N}$ il provided by Corollary 6.2.2 can be strict.

6.4.1 Example Consider the Eilenberg-MacLane space $K(\mathbb{F}, 2)$; the results of Anderson and Hodgkin imply that $K^{*}(K(\mathbb{F}, 2))$ identifies with $K^{*}(p t)$ [1]. Theorem 4.6 of [8] implies that the subalgebras

$$
H_{\overparen{E(1)}}^{*}(X) \text { and } H_{K U}^{*}(X)
$$

of $H^{*}(X)$ coincide, hence Corollary 6.2.2 applies and there is a monomorphism in $\mathcal{K} / \mathcal{N}$ il

$$
H_{K U}^{*}(K(\mathbb{F}, 2)) \hookrightarrow \alpha\langle 1\rangle H^{*}(K(\mathbb{F}, 2)) .
$$

This morphism does not induce an isomorphism in $\mathcal{K} / \mathcal{N} i l$. To see this, it is sufficient to show that $\beta\langle 1\rangle g^{\text {op }} H^{*}(K(\mathbb{F}, 2))$ is not trivial; this is straightforward.

6.4.2 Remark The Eilenberg-MacLane spaces provide universal examples of the failure of the $F$-monomorphism to be an $F$-isomorphism. It is this failure which provides an obstruction to extending the functor $H_{E}^{*}$ to an algebraic functor defined on $\mathcal{K}$. 


\section{Filtrations of functors and group cohomology}

This section reviews the results of [4] from the functorial point of view. This functorial presentation allows the generalization of these results to the case of Borel cohomology, which is presented in Section 8.

7.0.1 Notation Throughout this section, let $G$ be a finite group and let $n$ be a nonnegative integer.

\subsection{Quillen's theorem - the functorial approach}

This section gives an overview of the reinterpretation, due to Lannes, of Quillen's results on the algebraic variety associated to the cohomology of a finite group. For convenience, references are given in [12] rather than in the original sources. A further convenient reference is the section on group cohomology of [3].

Recall the definition of the Quillen category associated to a finite group $G$.

7.1.1 Definition Let $\mathcal{A}_{p}(G)$ denote the category with objects the elementary abelian $p$-subgroups of $G$ and morphisms the morphisms of groups which are composites of conjugation by an element of $G$ and the inclusion of subgroups.

7.1.2 Notation Let $\mathcal{C}_{p}^{G}$ denote the set of presheaves on the category $\mathcal{A}_{p}(G)$, namely functors $\mathcal{A}_{p}(G)^{\mathrm{op}} \rightarrow$ Set.

There is a forgetful functor $\mathcal{A}_{p}(G) \rightarrow \mathcal{E}^{\mathrm{f}}$, hence the set of homomorphisms between vector spaces induces a functor $\operatorname{Hom}(-,-): \mathcal{E}^{\mathrm{fop}} \times \mathcal{A}_{p}(G) \rightarrow$ Set. Let $F \in \mathcal{C}_{p}^{G}$ be a presheaf, then the product $F \times \operatorname{Hom}(-,-)$ induces a functor

$$
\mathcal{A}_{p}(G)^{\mathrm{op}} \times \mathcal{A}_{p}(G) \rightarrow \widehat{\mathcal{E}}^{\mathrm{f}},
$$

where $\widehat{\mathcal{E}}^{\mathrm{f}}$ denotes the category of functors $\mathcal{E}^{\mathrm{fop}} \rightarrow$ Set. If $F$ takes values in the category of finite sets, this defines an object of $\mathcal{G}^{\mathrm{op}}$.

7.1.3 Definition For $F \in \mathcal{C}_{p}^{G}$ a functor which takes values in finite sets, let $\int_{\mathcal{A}_{p}(G)} F$ be the functor in $\mathcal{G}^{\text {op }}$ which is the coend of the functor $F \times \operatorname{Hom}(-,-)$ [11, Chapter IX, Section 6]. Explicitly, this is the quotient of the functor

$$
V \mapsto \amalg_{E \in \mathcal{A}_{p}(G)} F(E) \times \operatorname{Hom}(V, E)
$$


by the quotient relation which is induced for morphisms $j: E_{1} \rightarrow E_{2}$ in $\mathcal{A}_{p}(G)$ by identifying the images of the morphisms

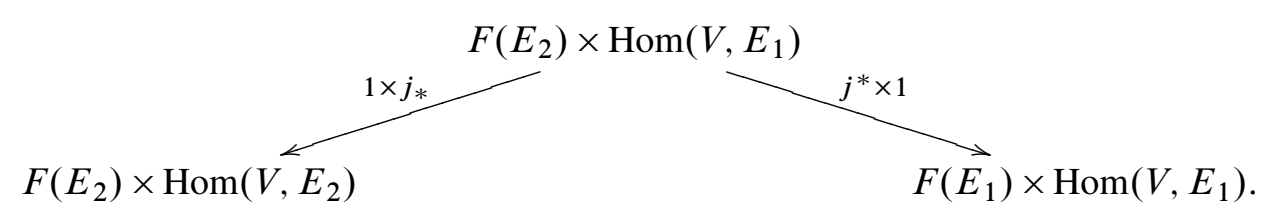

\subsubsection{Example}

(1) Let $F \in \mathcal{C}_{p}^{G}$ be the constant functor sending each $E \in \mathcal{A}_{p}(G)$ to a singleton set. The associated functor $\int_{\mathcal{A}_{p}(G)} F$ identifies with the colimit

$$
\underset{E \in \overrightarrow{\mathcal{A}_{p}}(G)}{\lim } \operatorname{Hom}(-, E) .
$$

This functor is isomorphic to the functor $V \mapsto \operatorname{Rep}(V, G)$, where $\operatorname{Rep}(V, G)$ is the set of group homomorphisms modulo the equivalence relation induced by conjugation by elements of $G$ [12, Corollary 3.8.8, Theorem 3.10.2].

(2) For $X$ a finite $G-\mathrm{CW}$-complex, let $F_{X}$ denote the functor sending $E \in \mathcal{A}_{p}(G)$ to the set $\pi_{0}\left(X^{E}\right) / C_{G}(E)$, where $C_{G}(E)$ denotes the centralizer of $E$ in $G$. The associated functor $\int_{\mathcal{A}_{p}(G)} F_{X}$ evaluated on $V \in \mathcal{E}^{\mathrm{f}}$ gives a set of equivalence classes with representatives of the form $(a, x)$, where $a$ is a morphism $V \rightarrow G$ and $x \in \pi_{0}\left(X^{\operatorname{Im}(a)}\right)$.

Quillen's theorem on the variety of the ring $H^{*}(B G)$ has the following interpretation in the context of unstable algebras.

7.1.5 Theorem For $G$ a finite group and $X$ a finite $G-C W$-complex, there are isomorphisms of functors in $\mathcal{G}^{\text {op }}$ :

$$
\begin{aligned}
g^{\mathrm{op}} H^{*}(B G) & \cong \operatorname{Rep}(-, G) ; \\
g^{\mathrm{op}} H^{*}\left(E G \times_{G} X\right) & \cong \int_{\mathcal{A}_{p}(G)} F_{X} .
\end{aligned}
$$

The second statement is equivalent to the description in terms of a limit over the Quillen category given in Proposition 7.1.7.

7.1.6 Definition For $F \in \mathcal{C}_{p}^{G}$ a functor $\mathcal{A}_{p}(G)^{\mathrm{op}} \rightarrow$ Set which takes values in finite sets, let $K(F) \in \mathcal{K}$ denote the unstable algebra over the Steenrod algebra given by

$$
K(F):=\operatorname{Hom}_{\mathcal{C}_{p}^{G}}\left(F, H^{*}(B-)\right),
$$


where $H^{*}(B-): E \mapsto H^{*}(B E)$ is regarded as a functor by forgetting the unstable algebra structure. (This definition is equivalent to defining a suitable end [11, Chapter IX, Section 5]). The structure of an unstable algebra is induced by the unstable algebra structure of $H^{*}(B E)$.

7.1.7 Proposition For $F \in \mathcal{C}_{p}^{G}$ a functor $\mathcal{A}_{p}(G)^{\mathrm{op}} \rightarrow$ Set which takes values in finite sets, there is an isomorphism of functors in $\mathcal{G}^{\text {op }}$

$$
g^{\mathrm{op}} K(F) \cong \int_{\mathcal{A}_{p}(G)} F .
$$

Proof This is a basic exercise using the definition of the functor $g: \mathcal{K} \rightarrow \mathcal{G}$, together with the definition of the respective end and coend. (See [12, Corollary 3.8.8]).

\subsection{Chern subrings for group cohomology}

Theorem 0.1 of [4] and Corollary 4.2 of [8] together imply the following result, in which $E(n)$ denotes the height- $n$ Johnson-Wilson spectrum.

7.2.1 Theorem For $G$ a finite group and $n$ a nonnegative integer, the unstable algebra $\mathrm{Ch}_{E(n)}^{*}(B G)$ has associated functor in $\mathcal{G}^{\mathrm{op}}$

$$
g^{\mathrm{op}} \mathrm{Ch}_{E(n)}^{*}(B G) \cong \beta\langle n\rangle \operatorname{Rep}(-, G) .
$$

Proof (Indications) Theorem 6.2.1 implies that there is a surjection in $\mathcal{G}^{\text {op }}$

$$
g^{\mathrm{op}} \mathrm{Ch}_{\overparen{E(n)}}^{*}(B G) \rightarrow \beta\langle n\rangle \operatorname{Rep}(-, G),
$$

and this surjection is an isomorphism [7]. The remainder of the proof consists of the comparison between $g^{\mathrm{op}} \mathrm{Ch}_{\overparen{E(n)}}^{*}(B G)$ and $g^{\mathrm{op}} \mathrm{Ch}_{E(n)}^{*}(B G)$ [8].

\subsection{Interpretation in terms of categories}

The following category, introduced by Green and Leary [5], generalizes the category $\mathcal{A}_{p}(G)$ of elementary abelian $p$-subgroups of $G$.

7.3.1 Definition Let $\mathcal{A}_{p}^{(n)}(G)$ denote the category with the same objects as $\mathcal{A}_{p}(G)$ and morphisms the inclusions $V \hookrightarrow W$ such that, for each morphism $\mathbb{F}^{n} \rightarrow V$, the restricted morphism between subgroups of $G$ is induced by conjugation by an element of $G$. 
The following Proposition is a generalization of the relation between the colimit over the Quillen category $\mathcal{A}_{p}(G)$ of $\operatorname{Hom}(-, W)$ and the functor $\operatorname{Rep}(-, G)$ :

7.3.2 Proposition For $G$ a finite group and $n \geq 0$ an integer, there is a natural isomorphism in $\mathcal{G}^{\text {op }}$

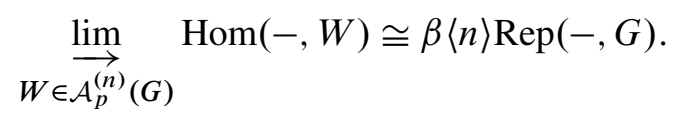

Proof (Indications) There is an embedding of categories $\mathcal{A}_{p}(G) \hookrightarrow \mathcal{A}_{p}^{(n)}(G)$ and the categories have the same objects, hence there is a surjection

$$
\operatorname{Rep}(-, G) \rightarrow \lim _{W \in \mathcal{A}_{p}^{(n)}(G)} \operatorname{Hom}(-, W) .
$$

It is straightforward to verify that this factors across the canonical surjection from $\operatorname{Rep}(-, G)$ onto $\beta\langle n\rangle \operatorname{Rep}(-, G)$.

In the other direction, for an object $W$ of $\mathcal{A}_{p}^{(n)}(G)$, there is an induced morphism $\operatorname{Hom}(-, W) \rightarrow \operatorname{Rep}(-, G) \rightarrow \beta\langle n\rangle \operatorname{Rep}(-, G)$. This morphism induces a morphism $\lim _{W \in \mathcal{A}_{p}^{(n)}(G)} \operatorname{Hom}(-, W) \rightarrow \beta\langle n\rangle \operatorname{Rep}(-, G)$.

These morphisms induce the required isomorphism of functors.

This recovers the main result of [4]:

7.3.3 Theorem For $G$ a finite group and $n \geq 0$ an integer, there exists a natural $F$-isomorphism

$$
\mathrm{Ch}_{E(n)}^{*}(B G) \rightarrow{\underset{W \in \mathcal{A}_{p}^{(n)}(G)}{\lim }} H^{*}(B W)
$$

\section{Borel cohomology}

Throughout this section, let $G$ be a finite group, let $X$ be a finite $G-\mathrm{CW}-$ complex, and let $n$ denote a fixed nonnegative integer. For clarity, the prime $p$ is suppressed from the notation.

Algebraic $8 \mathcal{G}$ Geometric Topology, Volume 6 (2006) 


\subsection{Generalized Chern rings for equivariant cohomology}

The arguments of Green, Hunton and Schuster [4] generalize to prove the following Theorem, using the generalized character theory of Hopkins, Kuhn and Ravenel [7]. There is an analogous result for the Chern subring, which is not stated here explicitly.

8.1.1 Theorem Let $E$ be a complex-oriented theory which satisfies Hypothesis 4.2.4 and the Hurewicz hypothesis $\mathbb{U}_{E}\left(B \mathbb{F}_{p}^{n}\right)$, for a nonnegative integer $n$. Then the unstable algebra $H_{E}^{*}\left(E G \times_{G} X\right)$ is $F$-isomorphic to $\alpha\langle n\rangle H^{*}\left(E G \times_{G} X\right)$.

Proof The proof is an application of Proposition 6.3.2 to the space $E G \times{ }_{G} X$, which involves the following steps:

(1) The Hurewicz morphism

$$
\left[B \mathbb{F}^{n}, E G \times_{G} X\right] \rightarrow \operatorname{Hom}_{\mathcal{K}}\left(H^{*}\left(E G \times_{G} X\right), H^{*}\left(B \mathbb{F}^{n}\right)\right)
$$

is surjective;

(2) The morphism which corresponds to $P_{H, E}$,

$$
g^{\mathrm{op}} H^{*}\left(E G \times_{G} X\right)\left(\mathbb{F}^{n}\right) \cong\left[B \mathbb{F}^{n}, E G \times_{G} X\right]_{H} \rightarrow\left[B \mathbb{F}^{n}, E G \times_{G} X\right]_{E},
$$

is injective.

These statements are proved in Section 8.2 and Section 8.4.

This provides the following generalization of Theorem 7.2.1 with respect to $E(n)$.

8.1.2 Corollary The unstable algebra $\mathrm{Ch}_{E(n)}^{*}\left(E G \times_{G} X\right)$ is $F$-isomorphic to $\alpha\langle n\rangle H^{*}\left(E G \times_{G} X\right)$, for $n$ a nonnegative integer.

Proof Theorem 8.1.1 applies to the complete Johnson-Wilson theory $\widehat{E(n)}$, by the results of [4] stated here in Example 4.1.3. Then Theorem 4.1 of [8] applies to replace the complete Johnson-Wilson theory by $E(n)$.

\subsection{Surjectivity of the Hurewicz morphism}

This section establishes that the Hurewicz morphism

$$
\left[B \mathbb{\mathbb { E }}^{n}, E G \times_{G} X\right] \rightarrow \operatorname{Hom}_{\mathcal{K}}\left(H^{*}\left(E G \times_{G} X\right), H^{*}\left(B \mathbb{\Vdash}^{n}\right)\right)
$$

is surjective and gives an explicit model for the right hand side. 
8.2.1 Definition Let $\mathcal{Y}_{n}$ denote the left $G$-, right $\operatorname{Aut}\left(\mathbb{F}^{n}\right)$-set with elements pairs $(\alpha,[x])$, where $\alpha: \mathbb{F}^{n} \rightarrow G$ is a group homomorphism and $[x] \in \pi_{0}\left(X^{\operatorname{Im}(\alpha)}\right)$. The left $G$-action is via conjugation on $\operatorname{Hom}\left(\mathbb{F}^{n}, G\right)$ and is induced via the restricted action $g: X^{\operatorname{Im}(\alpha)} \rightarrow X^{\operatorname{Im}\left(g \alpha g^{-1}\right)}$; the right $\operatorname{Aut}\left(\mathbb{F}^{n}\right)$-action is via the canonical action upon $\operatorname{Hom}\left(\mathbb{F}^{n}, G\right)$.

8.2.2 Lemma There is a map of sets $\mathcal{Y}_{n} \rightarrow\left[B \mathbb{F}^{n}, E G \times_{G} X\right]$ which sends a pair $(\alpha,[x])$ to the homotopy class of a morphism

$$
B \mathbb{F}^{n} \rightarrow B \mathbb{F}^{n} \times X^{\operatorname{Im}(\alpha)} \rightarrow E G \times_{G} X,
$$

where the first morphism is induced by $[x]$ and the second morphism is induced by $\alpha$.

Proof This is a standard geometric construction (see [7, Section 6]).

The set $\mathcal{Y}_{n}$ is related to the functor $F_{X}$ defined in Example 7.1.4 by the following result.

8.2.3 Proposition There is a canonical isomorphism of right Aut $\left(\mathbb{F}^{n}\right)-$ sets

$$
\left\{\int_{\mathcal{A}_{p}(G)} F_{X}\right\}\left(\mathbb{F}^{n}\right) \cong \mathcal{Y}_{n} / G
$$

Proof An object of $\mathcal{A}_{p}(G)$ is an elementary abelian $p$-group $V$ equipped with a monomorphism $V \hookrightarrow G$. There is a map $F_{X}(V) \times \operatorname{Hom}\left(\mathbb{F}^{n}, V\right) \rightarrow \mathcal{Y}_{n}$ which is induced by composition with $V \hookrightarrow G$. It is straightforward to verify that this induces a surjective morphism

$$
\left\{\int_{\mathcal{A}_{p}(G)} F_{X}\right\}\left(\mathbb{F}^{n}\right) \rightarrow \mathcal{Y}_{n} / G
$$

and that this is a monomorphism.

8.2.4 Corollary The Hurewicz morphism is surjective and there are bijections

$$
g^{\text {op }} H^{*}\left(E G \times_{G} X\right)\left(\mathbb{F}^{n}\right) \cong \mathcal{Y}_{n} / G \cong\left[B \mathbb{F}^{n}, E G \times_{G} X\right]_{H} .
$$

\subsection{Hopkins-Kuhn-Ravenel generalized character theory}

This section exploits the work of Hopkins, Kuhn and Ravenel on generalized group characters; for the convenience of the reader, a rapid review of the theory is included. Refer to the original source [7] for the details.

For $E$ a complex-oriented theory satisfying Hypothesis 4.2.4, the ring $L\left(E^{*}\right)$ is the localization of the continuous cohomology $E_{\text {cont }}^{*}\left(B \mathbb{Z}_{p}^{n}\right)$ inverting the Euler classes 
corresponding to continuous morphisms $\mathbb{Z}_{p}^{n} \rightarrow S^{1}$ [7]. There is a natural action of the group $\operatorname{Aut}\left(\mathbb{Z}_{p}^{n}\right)$ of continuous automorphisms upon $L\left(E^{*}\right)$.

The following result contains the fundamental faithful-flatness property of the ring $L\left(E^{*}\right)$, which allows the simplification of arguments by using faithfully flat descent.

8.3.1 Proposition [7] The ring $L\left(E^{*}\right)$ satisfies the following properties:

(1) $L\left(E^{*}\right)$ is a faithfully flat $\left(p^{-1} E^{*}\right)$-module;

(2) the ring of invariants $L\left(E^{*}\right)^{\operatorname{Aut}\left(\mathbb{Z}_{p}^{n}\right)}$ is isomorphic to $p^{-1} E^{*}$;

(3) there is an isomorphism of $L\left(E^{*}\right)$-modules

$$
L\left(E^{*}\right) \otimes_{E^{*}} E^{*}\left(B \mathbb{\Vdash}^{k}\right) \cong \amalg_{\mathrm{Hom}\left(\mathbb{F}^{n}, \mathbb{F}^{k}\right)} L\left(E^{*}\right) .
$$

Let $\operatorname{Fix}_{n}(G, X)$ denote the $G \times \operatorname{Aut}\left(\mathbb{Z}_{p}^{n}\right)$-space $\amalg_{a \in \operatorname{Hom}\left(\mathbb{Z}_{p}^{n}, G\right)} X^{\operatorname{Im}(a)}$. The ring of generalized class functions is

$$
C l_{n}\left(G, X ; L\left(E^{*}\right)\right):=L\left(E^{*}\right) \otimes_{E^{*}} E^{*}\left(\operatorname{Fix}_{n}(G, X)\right)^{G},
$$

which is an $L\left(E^{*}\right)$-algebra with an $\operatorname{Aut}\left(\mathbb{Z}_{p}^{n}\right)$-action. The generalized character map is the morphism of $E^{*}$-algebras

$$
\chi_{n}^{G}: E^{*}\left(E G \times_{G} X\right) \rightarrow C l_{n}\left(G, X ; L\left(E^{*}\right)\right)^{\operatorname{Aut}\left(\mathbb{Z}_{p}^{n}\right),}
$$

which is induced by the product of the morphisms $B\left(\mathbb{Z} / p^{m}\right)^{n} \times X^{\operatorname{Im}(a)} \rightarrow E G \times_{G} X$ induced by $a:\left(\mathbb{Z} / p^{m}\right)^{n} \rightarrow G$.

8.3.2 Theorem [7, Theorem C] For $E$ a complex-oriented theory satisfying Hypothesis 4.2.4, the generalized character map $\chi_{n}^{G}$ induces isomorphisms:

$$
\begin{aligned}
& p^{-1} E^{*}\left(E G \times_{G} X\right) \stackrel{\cong}{\longrightarrow} C l_{n}\left(G, X ; L\left(E^{*}\right)\right)^{\operatorname{Aut}\left(\mathbb{Z}_{p}^{n}\right) ;} \\
& L\left(E^{*}\right) \otimes_{E^{*}} E^{*}\left(E G \times_{G} X\right) \stackrel{\cong}{ } C l_{n}\left(G, X ; L\left(E^{*}\right)\right) .
\end{aligned}
$$

\subsection{Separation}

Throughout this section $E, G$ and $X$ satisfy the hypotheses of Theorem 8.3.2.

It is necessary to show that the map $\left[B \mathbb{F}^{n}, E G \times_{G} X\right]_{H} \rightarrow\left[B \mathbb{F}^{n}, E G \times_{G} X\right]_{E}$ is injective; this is equivalent to showing that the map $\gamma$ in the diagram

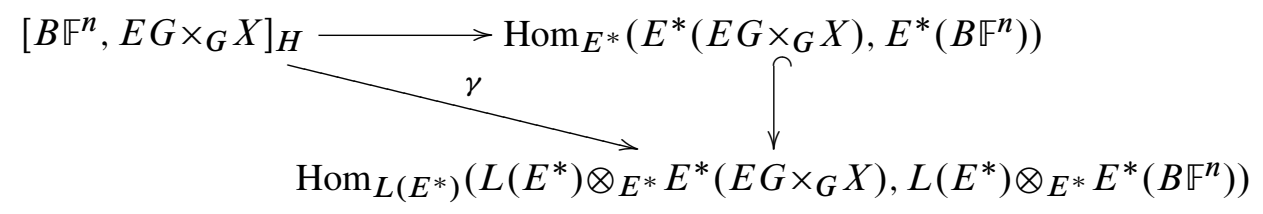

Algebraic $\&$ Geometric Topology, Volume 6 (2006) 
is injective, since the vertical morphism is an injection, by faithful flatness of $L\left(E^{*}\right)$ over $E^{*}$.

The set $\left[B \mathbb{E}^{n}, E G \times_{G} X\right]_{H}$ identifies with $\mathcal{Y}_{n} / G$ by Corollary 8.2 .4 and the ring $L\left(E^{*}\right) \otimes_{E^{*}} E^{*}\left(E G \times_{G} X\right)$ identifies with $C l_{n}\left(G, X ; L\left(E^{*}\right)\right)$ by Theorem 8.3.2.

8.4.1 Lemma There is a surjection

$$
C l_{n}\left(G, X ; L\left(E^{*}\right)\right) \rightarrow L\left(E^{*}\right) \otimes_{E^{*}} E^{*}\left(\mathcal{Y}_{n}\right)^{G}
$$

which is induced by restriction to elementary abelian subgroups and the passage to path-connected components.

Proof The inclusion of the subspace $\amalg_{a \in \operatorname{Hom}\left(\mathbb{F}^{n}, G\right)} X^{\operatorname{Im}(a)}$ of $\operatorname{Fix}_{n}(G, X)$ induces a surjection $E^{*}\left(\operatorname{Fix}_{n}(G, X)\right) \rightarrow E^{*}\left(\amalg_{a \in \operatorname{Hom}\left(\mathbb{F}^{n}, G\right)} X^{\operatorname{Im}(a)}\right)$, which is a morphism of $G$-modules.

There is a morphism of spaces $\mathcal{Y}_{n} \rightarrow \amalg_{a \in \operatorname{Hom}\left(\mathbb{F}^{n}, G\right)} X^{\operatorname{Im}(a)}$ induced by choice of basepoint in each path-connected component. The morphism has a retract provided by the natural projection $1 \rightarrow \pi_{0}$ and it preserves the $G$-action up to homotopy; hence the morphism gives rise to a surjection

$$
E^{*}\left(\amalg_{a \in \operatorname{Hom}\left(\mathbb{F}^{n}, G\right)} X^{\operatorname{Im}(a)}\right) \rightarrow E^{*}\left(\mathcal{Y}_{n}\right)
$$

of $G$-modules.

The composite induces the required surjection, either by constructing a section as a morphism of $G$-modules or by using the exactness of the $G$-fixed point functor, as in the proof of [7, Theorem $\mathrm{C}]$.

8.4.2 Remark The $L\left(E^{*}\right)$-module $L\left(E^{*}\right) \otimes_{E^{*}} E^{*}\left(\mathcal{Y}_{n}\right)$ is a permutation $G$-module, free on basis indexed by the elements of $\mathcal{Y}_{n}$. It follows that the $G$-invariants form a free $L\left(E^{*}\right)$-module indexed over the orbits $\mathcal{Y}_{n} / G$.

8.4.3 Lemma The morphism $\gamma$ factorizes as

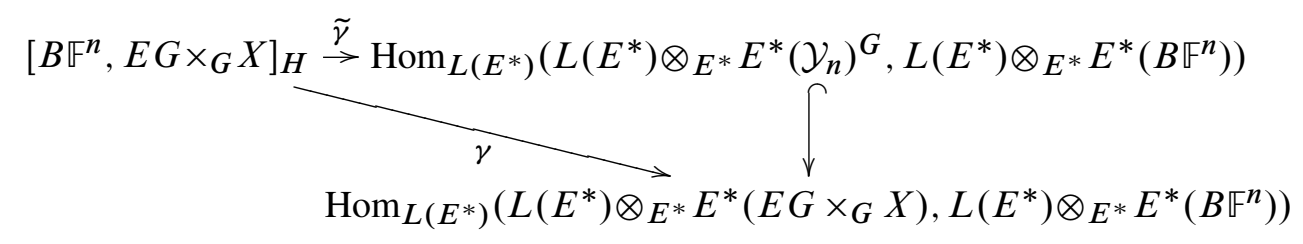

in which the inclusion is induced by the morphism of Lemma 8.4.1. 
Proof The result follows from the definition of the generalized character map.

Hence, the proof is completed by the following result.

8.4.4 Proposition The morphism $\tilde{\gamma}$ is a monomorphism.

Proof The $L\left(E^{*}\right)$-module $L\left(E^{*}\right) \otimes_{E^{*}} E^{*}\left(\mathcal{Y}_{n}\right)^{G}$ is a free $L\left(E^{*}\right)$-module on basis indexed by the orbits $\mathcal{Y}_{n} / G$. For $\bar{y}$ an orbit of $\mathcal{Y}_{n} / G$, the morphism $\tilde{\gamma}(\bar{y})$ is nontrivial and factors across the projection onto the direct summand indexed by $\bar{y}$. The required injectivity follows.

\section{References}

[1] D W Anderson, L Hodgkin, The $K$-theory of Eilenberg-MacLane complexes, Topology 7 (1968) 317-329 MR0231369

[2] A Baker, U Würgler, Bockstein operations in Morava K-theories, Forum Math. 3 (1991) 543-560 MR1129998

[3] W G Dwyer, H-W Henn, Homotopy theoretic methods in group cohomology, Advanced Courses in Mathematics. CRM Barcelona, Birkhäuser Verlag, Basel (2001) MR1926776

[4] D J Green, J R Hunton, B Schuster, Chromatic characteristic classes in ordinary group cohomology, Topology 42 (2003) 243-263 MR1928652

[5] D J Green, I J Leary, The spectrum of the Chern subring, Comment. Math. Helv. 73 (1998) 406-426 MR1633363

[6] H-W Henn, J Lannes, L Schwartz, The categories of unstable modules and unstable algebras over the Steenrod algebra modulo nilpotent objects, Amer. J. Math. 115 (1993) 1053-1106 MR1246184

[7] M J Hopkins, N J Kuhn, D C Ravenel, Generalized group characters and complex oriented cohomology theories, J. Amer. Math. Soc. 13 (2000) 553-594 MR1758754

[8] J R Hunton, B Schuster, Subalgebras of group cohomology defined by infinite loop spaces, Topology 44 (2005) 689-704 MR2136531

[9] N J Kuhn, Generic representations of the finite general linear groups and the Steenrod algebra. I, Amer. J. Math. 116 (1994) 327-360 MR1269607

[10] J Lannes, Sur les espaces fonctionnels dont la source est le classifiant d'un p-groupe abélien élémentaire, Inst. Hautes Études Sci. Publ. Math. (1992) 135-244 MR1179079

[11] S Mac Lane, Categories for the working mathematician, second edition, Graduate Texts in Mathematics 5, Springer, New York (1998) MR1712872 
[12] L Schwartz, Unstable modules over the Steenrod algebra and Sullivan's fixed point set conjecture, Chicago Lectures in Mathematics, University of Chicago Press, Chicago, IL (1994) MR1282727

Laboratoire Analyse, Géométrie et Applications, UMR 7539, Institut Galilée

Université Paris 13, 93430 Villetaneuse, France

powell@math . univ-paris13.fr

http://www . math. univ-paris13.fr/ powell

Received: 7 July 2005

Algebraic $\&$ Geometric Topology, Volume 6 (2006) 